\title{
Noncommutativity and Lorentz violation in relativistic heavy ion collisions
}

\author{
P. Castorina ${ }^{1,2, a}$, A. Iorio $^{3}$, D. Zappalà ${ }^{2}$ \\ ${ }^{1}$ Dipartimento di Fisica, Università di Catania, Via Santa Sofia 64, 95123 Catania, Italy \\ ${ }^{2}$ INFN, Sezione di Catania, 95123 Catania, Italy \\ ${ }^{3}$ Faculty of Mathematics and Physics, Charles University in Prague, V. Holešovičkách 2, 18000 Prague 8, Czech Republic
}

Received: 25 January 2011 / Revised: 22 March 2011 / Published online: 31 May 2011

(C) The Author(s) 2011. This article is published with open access at Springerlink.com

\begin{abstract}
We show that relativistic heavy ion collisions at LHC energies could be used as an experimental probe to detect fundamental properties of spacetime long speculated about. Our results rely on the recent proposal that magnetic fields of intensity much larger than that of magnetars should be produced at the beginning of the collisions and this could have an important impact on the experimental manifestation of a noncommutative spacetime. Indeed, in the noncommutative generalization of electrodynamics the interplay between a nonzero noncommutative parameter and an external magnetic field leads us to predict the production of lepton pairs of low invariant mass by free photons (an event forbidden by Lorentz invariant electrodynamics) in relativistic heavy ion collisions at present and future available energies. This unique channel can be clearly considered as a signature of noncommutativity. On the other hand, the search for such decays is worth anyway because their absence would ameliorate of three orders of magnitude the current bound on the noncommutative parameter.
\end{abstract}

High energy heavy ion collisions are a powerful experimental tool to investigate new fundamental physics. At the Relativistic Heavy Ion Collider (RHIC) and even more at the Large Hadron Collider (LHC) we shall be able to probe further solidly tested theories, such as Quantum Chromo Dynamics (QCD) and the whole Standard Model of particle physics. We also hope to have within that experimental reach some signatures of the elusive effects of to-date fully speculative theories, such as, e.g., supersymmetry. In this letter we intend to show that, due to recent results, heavy ion collisions should also be used for the experimental search of noncommutative properties of our spacetime.

a e-mail: paolo.castorina@ct.infn.it
It has been recently shown [1-4] that fundamental aspects of QCD related to the topological nature of its vacuum can be directly tested by experimental observations in relativistic heavy ion collisions. Gluon field configurations with nonzero topological charge generate chiral asymmetry, inducing $P$ and $C P$ violating effects which produce an asymmetry between the amount of positive and negative charge above and below the reaction plane [1-4]. STAR Collaboration presented $[5,6]$ the conclusive observation of chargedependent azimuthal correlations, however the explanation of this charge asymmetry by $P$ - and $C P$-odd dynamics requires that a strong magnetic field is produced at the beginning of the collision. ${ }^{1}$ Analytical calculations [3] and numerical simulations [10] show that it is possible to produce an extremely intense magnetic field $|B| \simeq m_{\pi}^{2}$ in peripheral heavy ion collisions at RHIC. We shall show here that the production of magnetic fields of such intensity could open a window on the detection of fundamental properties of spacetime through the manifestation of the effects of noncommutativity of coordinates [11-13] and the associated violation of Lorentz symmetry [14-16].

Besides noncommutativity, many other mechanisms for Lorentz violation exist and have been proposed in: the Standard Model Extension [17], theories with speed of light differing from $c$ and various string theory/quantum gravityinspired effective field theories [18-21]. In all cases, including noncommutative field theories, the bounds on the Lorentz violating parameters make the experimental appreciation extremely elusive. ${ }^{2}$ Here we choose the noncommu-

\footnotetext{
${ }^{1}$ For different explanations see [7-9].

${ }^{2}$ For example, a low energy remnant of quantum gravity is posited to be a modification of the dispersion relation for the photon given by, $(c=1), E^{2}-k^{2}=-\xi k^{3} / M_{P l}$ where $M_{P l} \simeq 10^{19} \mathrm{GeV}$ is the Planck mass and $\xi$ is a parameter that should be smaller than $10^{-15}$, see, e.g., [22].
} 
tative framework of Lorentz violation because in the presence of a strong magnetic field it is the one that allows for a very clear prediction of a unique decay channel. That the quantum phase of these theories may [23] or may not [24] be affected by certain novel divergencies is not of concern to us here, because we shall focus on the kinematical bounds to quantum processes and to this end classical considerations are perfectly sound (see, e.g., [25-29]).

In the canonical formulation of spacetime noncommutativity one has $x^{\mu} \star x^{\nu}-x^{\nu} \star x^{\mu}=i \theta^{\mu \nu}$, with $\star$ the MoyalWeyl product, $\theta^{\mu \nu}$ an antisymmetric constant tensor (see, e.g., [30]) and $\mu, v=0,1,2,3$. The general recipe to deal with gauge theories in such spacetimes was given in [31]. We are interested here on the $O(\theta)$-corrected action for Maxwell theory given by

$$
\begin{aligned}
\hat{I}= & -\frac{1}{4} \int d^{4} x\left[F^{\mu \nu} F_{\mu \nu}-\frac{1}{2} \theta^{\alpha \beta} F_{\alpha \beta} F^{\mu \nu} F_{\mu \nu}\right. \\
& \left.+2 \theta^{\alpha \beta} F_{\alpha \mu} F_{\beta \nu} F^{\mu \nu}\right],
\end{aligned}
$$

with $F_{\mu \nu}=\partial_{\mu} A_{\nu}-\partial_{\nu} A_{\mu}$, and $A_{\mu}$ the usual Abelian gauge field. Clearly, noncommutative electrodynamics (NCED) is essentially a nonlinear generalization of Maxwell electrodynamics and it was shown [25] that plane waves exist and, while those propagating along the direction of a background magnetic field $\boldsymbol{B}$ still travel at the usual speed of light, those which propagate transversely to $\boldsymbol{B}$ have a modified dispersion relation given by

$\omega=k\left(1-\boldsymbol{\theta}_{T} \cdot \boldsymbol{B}_{T}\right)$

where $\boldsymbol{\theta} \equiv\left(\theta^{1}, \theta^{2}, \theta^{3}\right)$ is the spatial part of $\theta^{\mu \nu}\left(\theta^{i}=\right.$ $\frac{1}{2} \epsilon^{i j k} \theta^{j k}, i, j, k=1,2,3$, the temporal components are taken to be zero $\theta^{0 i}=0$ ) and the subscript $T$ indicates the transverse component with respect to $\boldsymbol{k}$. NCED has been discussed by considering synchrotron radiation [26], Čerenkov effect in vacuum [27], ultra high energy gamma rays [28, 29]. In all those works it was concluded that the effects of a nonzero $\boldsymbol{\theta}$ in NCED are very hard to detect due to the actual upper bound $\theta_{\text {bound }} \simeq 1 /(10 \mathrm{TeV})^{2}[14],{ }^{3}$ and to the unavailability in nature of intense enough magnetic fields.

In the initial state of relativistic heavy ion collisions, for large impact parameters, the magnetic field at the center of the collision can be approximately written as $[3,10,33]$

$B(t)=\frac{1}{\left[1+(t / \tau)^{2}\right]^{3 / 2}} B_{0}$,

with $\tau=b /(2 \sinh Y), B_{0}=8 Z \alpha_{\mathrm{EM}} \sinh Y / b^{2}$ where $b$ denotes the impact parameter, $Z$ the charge of the nucleus,

\footnotetext{
${ }^{3}$ For a bound on noncommutative parameters in phase space see [32].
}

and $Y$ the beam rapidity. For gold-gold $(Z=79)$ collisions at RHIC (at $100 \mathrm{GeV}$ per nucleon) one has $Y=5.36$ and, at typical large impact parameters $b=10 \mathrm{fm}$, one finds $B_{0} \sim 1.9 \times 10^{5} \mathrm{MeV}^{2}$ and $\tau=0.05 \mathrm{fm}$.

From this it appears that the initial magnetic field in relativistic heavy ion collisions is much larger than the magnetic fields of magnetars and one can consider the possibility of detection of the Lorentz violating nonzero $\boldsymbol{\theta}$ effects. With these extremely intense magnetic fields one gains for $\left|\boldsymbol{\theta}_{T} \cdot \boldsymbol{B}_{T}\right|$ more than 20 orders of magnitude with respect to magnetic fields in terrestrial laboratories.

Before the evaluation of the impact of such a gain on the dispersion relations in (2), which will be our main point here, let us give first an estimate of the impact on the noncommutative corrections to the synchrotron radiation spectrum of an energetic quark traveling in the magnetic field produced at the beginning of the collision.

The synchrotron radiation spectrum in NCED has been evaluated in [26]. Let us call $\omega$ the radiation frequency, $\omega_{0} \sim 1 /|\boldsymbol{r}|$ the cyclotron frequency, $\omega_{c}=3 \omega_{0} \gamma^{3}$ the critical frequency, where $\boldsymbol{r}$ is the radius of the orbit and $\gamma$ the Lorentz factor. In the range $1 \ll \omega / \omega_{0} \ll \gamma^{3}$, the ratio of the $\theta$-corrected energy $I$ (radiated in the plane of the orbit at large distances from the origin, within an angle $d \Omega$ ) to the standard one $I_{\theta=0}$, in the most favorable case $\boldsymbol{\theta}_{T} \| \boldsymbol{B}_{T}$, is given by

$X \equiv \frac{d I(\omega) / d \Omega}{d I(\omega) /\left.d \Omega\right|_{\theta=0}} \sim 1+20\left(\frac{\omega_{0}}{\omega}\right)^{2 / 3}|\theta B| \gamma^{4}$.

For light energetic valence quarks in the heavy ion beams of $100 \mathrm{GeV}$ per nucleon the Lorentz factor could easily be $\gamma \simeq O\left(10^{2}-10^{3}\right)$ and by considering $\omega_{0} \simeq 1 / \tau$, where $\tau$ has been introduced in (3), the range $1 \ll \omega / \omega_{0} \ll \gamma^{3}$ allows the production of high frequency radiation. With $|\theta B| \simeq 10^{-9}$, for gold-gold collisions at RHIC, the ratio $X$ can be larger than 1 indicating that either the spectrum is the $\theta$-corrected or, conversely, that the $\theta_{\text {bound }}$ used must be ameliorated. We do not proceed further with this case because to go beyond a mere indication of the effect here we should consider the situation in greater detail (higher order contributions, timevarying magnetic fields, parton distribution of the initial nucleon momentum, etc.).

What we can instead reliably focus on here are the dispersion relations in (2) regarded as the kinematical threshold for event obviously forbidden in standard electrodynamics, i.e. the pair production from a single free photon $\gamma \rightarrow e^{+} e^{-}$, independently on the prompt photon production mechanism. It is essential to note that the modified dispersion relation (2), as obtained in [25], is read from the exact solution of the field equations coming from the noncommutative Lagrangian in (1). In other words, the effect of the tri-linear term $O\left(F^{3}\right)$ in (1) is completely included. Moreover, for dimensional reasons, according to our Lagrangian in (1) any 
possible perturbative correction to a cross section should be of order $O\left((\theta B)^{2}\right)$ and, since in our case $|\theta B| \simeq 10^{-9}$, the higher order terms are negligible.

In (2) the noncommutative contribution depends on the angle between $\boldsymbol{B}_{T}$ and $\boldsymbol{\theta}_{T}$. At first order in $\theta$ one has

$\omega\left(1+\boldsymbol{\theta}_{T} \cdot \boldsymbol{B}_{T}\right)=k$

or

$E_{\gamma}^{2}-k^{2}=-2 E_{\gamma}^{2}\left(\boldsymbol{\theta}_{T} \cdot \boldsymbol{B}_{T}\right)$,

where $E_{\gamma} \equiv \omega$.

Defining by $p_{\gamma}, p_{+}$and $p_{-}$the four momenta of $\gamma$, of $e^{+}$and of $e^{-}$, respectively, the kinematical condition for the decay of a free photon whose action is given by (1) is

$-2 E_{\gamma}^{2}\left(\boldsymbol{\theta}_{T} \cdot \boldsymbol{B}_{T}\right)=\left(p_{+}+p_{-}\right)^{2}>4 m_{e}^{2}$

which requires $\left(\boldsymbol{\theta}_{T} \cdot \boldsymbol{B}_{T}\right)<0$. Therefore if in a heavy ion collision at RHIC one produces a magnetic field of about $10^{5} \mathrm{MeV}^{2}$, with $\theta \simeq \theta_{\text {bound }}$, a free photon can produce an $e^{+} e^{-}$pair. Hence an enhancement of lepton pairs with low invariant mass could be the signal of the "exotic" effects of noncommutativity. With a magnetic field $B \sim 2 \times 10^{5} \mathrm{MeV}^{2}$ a photo with energy $E_{\gamma}=50 \mathrm{GeV}$ (and then with typical time scale $\tau_{\gamma} \equiv 1 / E_{\gamma} \sim 4 \times 10^{-3} \mathrm{fm}$ ) opens a new channel for lepton pairs of invariant mass of about $3 \mathrm{MeV}$, while for $B \sim 2 \times 10^{6} \mathrm{MeV}^{2}$ and $E_{\gamma}=100 \mathrm{GeV}$ (hence $\tau_{\gamma} \sim$ $2 \times 10^{-3} \mathrm{fm}$ ) the invariant mass is about $20 \mathrm{MeV}$.

Of course lepton pairs of different origins are abundant in heavy ion collisions [34] and the rest of this letter is dedicated to single out the nonzero $\boldsymbol{\theta}$ effect from this large background. To this end we first notice that only $\gamma$ s produced at the very beginning of the collisions have to be considered because the magnetic field rapidly decreases with time (see (3)) and noncommutative effects, if any, fade away accordingly. For $E_{\gamma}=50 \mathrm{GeV}$ or $E_{\gamma}=100 \mathrm{GeV}$, however, we have

$\tau_{\gamma} \ll \tau$

hence the magnetic field is enough strong for long enough in these cases. Those high energy $\gamma \mathrm{s}$ should produce pairs of very small invariant mass (see (7)). This implies that the electron and positron are essentially produced collinearly but, since such a "exotic" decay occurs at the very beginning of the collision, one should observe the $e^{+}$and $e^{-}$with very large energy and an initial opposite curvature. Thus this pair production is completely different from: (a) the standard Drell-Yan process; (b) resonances decay and (c) the usual $\gamma \gamma \rightarrow e^{-} e^{+}$and/or the pair production in the electromagnetic field, because in all the above cases one has large invariant masses and/or mainly forward production [34].
We proceed now to single out the effects of the magnetic field. The first step is to consider the ratio between the yields of low invariant mass lepton pairs in nucleus-nucleus (A-B) and proton-proton $(\mathrm{p}-\mathrm{p})$ collisions because in the latter the collective magnetic field is negligible [3]. However, in heavy ion collisions rescattering effects still generate a significant background and therefore we need to go further. Defining the laboratory frame, say $(\hat{x}, \hat{y}, \hat{z})$, in such a way that the reaction plane corresponds to the $\hat{x}-\hat{y}$ plane, the magnetic field $B$ is produced in the $\hat{z}$ direction [3]. The noncommutative effect is enhanced if the magnetic field transverse to the direction of the momentum of the photon is maximum (see (7)) and then one has to focus on the reaction plane. In particular, if $\hat{y}$ is the beam axis, to avoid the background in the forward direction, it is convenient to consider the $\gamma$ production in the reaction plane and with large transverse momentum, as when the prompt photon is produced by an initial Compton-like quark-gluon $\rightarrow$ quark $-\gamma$ scattering, see, e.g., [35] (on this more later). For instance, if $\boldsymbol{k} \simeq\left(k_{x}, 0,0\right)$ the noncommutative effect depends on the product $\theta_{z} B$. Thus a clear signal would be an enhancement, event by event, of pairs of low invariant mass in the reaction plane with respect those produced outside the reaction plane.

Since the previous asymmetry varies event by event, according to the angle between $\boldsymbol{\theta}$ and $\boldsymbol{B}$, one should in principle rely experimentally on correlation techniques (see, e.g., [36] and references therein). Analogously to the analysis of the STAR collaboration $[5,6]$ on the charge asymmetry in peripheral collisions, in our case one has to consider correlations among photons (or electron-positron pairs) with a fixed azimuth relative to the reaction plane. As well known [36], if one defines by $\phi_{1}$ and $\phi_{2}$ the azimuth of two photons (two electron-positron pairs), the correlator $\left\langle\cos \left(\phi_{1}+\right.\right.$ $\left.\left.\phi_{2}-2 \Psi_{R P}\right)\right\rangle$, where $\Psi_{R P}$ is the angle of the reaction plane in the laboratory frame, must be zero if there is the up-down symmetry. Thus a further indication, although difficult to detect due to low statistics, of noncommutativity is a nonzero correlator.

Let us now go back to the low mass $e^{+} e^{-}$production at large transverse momentum to give a rough estimate, for relativistic heavy ion at $\mathrm{LHC}$, of the noncommutative contribution with respect to the other sources: the Compton-like quark-gluon scattering where a quasi real photon $\gamma^{*}$ decays in $e^{+} e^{-}$, and the Dalitz decay, $\pi^{0} \rightarrow \gamma e^{+} e^{-}$where $\pi^{0}$ are produced via gluon scattering.

In the kinematical region we are considering,the contribution due to $\gamma^{*} \rightarrow e^{+} e^{-}$can be evaluated by the differential cross section for prompt real photon times the kinematical factor $\alpha \ln \left[M_{\max }^{2} /\left(4 m_{e}^{2}\right)\right] / 3 \pi[35,37]$ where $M_{\max }$ is the largest invariant mass allowed. The same method can be applied to evaluate the $e^{+} e^{-}$pairs produced by the decay of noncommutative photons (there are only minor perturbative, noncommutative, correction to the differential cross section; 
the main effect is the modification of the kinematical threshold).

Concerning the $\pi^{0}$ contribution, its differential cross section can be evaluated by taking into account the branching ratio $B\left(\pi^{0} \rightarrow \gamma e^{+} e^{+} / \pi^{0} \rightarrow \gamma \gamma\right) \simeq 0.012$ [38] and that for $p_{T}^{\gamma} \geq 50 \mathrm{GeV}$ the $\pi^{0} \rightarrow \gamma \gamma$ differential cross section, for $\mathrm{Pb}-\mathrm{Pb}$ collision at $\sqrt{s}=5.5 \mathrm{TeV}$, is essentially the same as the prompt real photon one [37].

Therefore the total differential cross section includes three contributions, the two standard background (SB) ones just mentioned and the noncommutative one, and the ratio noncommutative signal to SB can be written as $N_{\text {tot }} / N_{\mathrm{SB}}=$ $1+K_{\mathrm{NC}}$ where $N$ is the number of $e^{+} e^{-}$pairs and $K_{\mathrm{NC}}$ is given by

$K_{\mathrm{NC}}=\frac{\frac{\alpha}{3 \pi} \ln \left(\frac{2 E_{\gamma}^{2}\left(\boldsymbol{\theta}_{T} \cdot \boldsymbol{B}_{T}\right)}{4 m_{e}^{2}}\right)}{0.012+\frac{\alpha}{3 \pi} \ln \left(\frac{M_{\max }^{2}}{4 m_{e}^{2}}\right)}$,

At $E_{\gamma}=p_{T}^{\gamma}=50 \mathrm{GeV}, B \simeq 1.9 \times 10^{5} \mathrm{MeV}^{2}$, and $2 E_{\gamma}^{2}\left(\boldsymbol{\theta}_{T}\right.$. $\left.\boldsymbol{B}_{T}\right)=10 \mathrm{MeV}^{2}=M_{\mathrm{max}}^{2}$, (9) gives a correction of about 13 per cent. At $E_{\gamma}=p_{T}^{\gamma}=100 \mathrm{GeV}, B \simeq 3 \times 10^{6} \mathrm{MeV}^{2}$, $2 E_{\gamma}^{2}\left(\boldsymbol{\theta}_{T} \cdot \boldsymbol{B}_{T}\right)=400 \mathrm{MeV}^{2}=M_{\max }^{2}$ and the correction is about 27 per cent. In the transverse momentum region 80-100 GeV, the differential cross section for prompt photons production $E d \sigma / d^{3} p$ is equal to the $\pi^{0} \rightarrow \gamma \gamma$ one: $\simeq 10^{-6} \mathrm{mb} / \mathrm{GeV}^{2}$ [37]. Therefore a rough estimate of the number of events, including the three contributions, can be done by considering $B \simeq 3 \times 10^{6} \mathrm{MeV}^{2}, d \sigma / d p_{T} d y \mid y=$ $0 \simeq\left(2 \pi p_{T}\right) E d \sigma / d^{3} p$ ( $y$ is the photon or pion rapidity) and following our previous discussion. For a LHC luminosity for heavy ion collisions, $L \simeq 5 \times 10^{26} \mathrm{~cm}^{-2} \mathrm{sec}^{-1}$ [37] and for a running time $\simeq 10^{6} \mathrm{sec}$, one expects about 100 events with about 25 events due to the noncommutative contribution. The latter estimate is obtained for $\boldsymbol{\theta}_{T} \cdot \boldsymbol{B}_{T}=\theta_{z} \boldsymbol{B}$ and by using the value $\theta_{z} \sim 1 /(10 \mathrm{TeV})^{2}$. In this sense it has to be considered as an upper bound on the effective yield due to the noncommutative effects.

On the other hand, $\boldsymbol{\theta}$ is fixed in a non-rotating frame, denoted by $(\hat{X}, \hat{Y}, \hat{Z})$, whereas the component $\theta_{z}$ used above is defined in the previously introduced frame. Since this frame, at fixed reaction plane, rotates with the earth, this component changes in time with the periodicity that depends on the earth's sidereal rotation frequency $\Omega$. By following the choice in $[14,39]$, one can take the $\hat{Z}$ direction of the nonrotating frame coincident with the rotation axis of the earth and $\hat{X}$ and $\hat{Y}$ with specific fixed celestial equatorial coordinates. Then, by indicating with $\left(\theta_{X}, \theta_{Y}, \theta_{Z}\right)$ the components of the noncommutative parameter in the non-rotating frame, one gets the explicit time dependence of $\theta_{z}$ [39]

$\theta_{z}=(\sin \chi \cos \Omega t) \theta_{X}+(\sin \chi \sin \Omega t) \theta_{Y}+\cos \chi \theta_{Z}$, where $\chi$ is the non-vanishing time-independent angle between the two axes $\hat{Z}$ and $\hat{z}$. The oscillation in $\theta_{z}$ disappears in the peculiar case of $\boldsymbol{\theta}$ coincident with the earth rotation axis (i.e. $\boldsymbol{\theta}=\theta_{Z}$ and therefore $\theta_{z}=\cos \chi|\boldsymbol{\theta}|$ ) whereas it is maximal if $\boldsymbol{\theta}$ lies in the equatorial plane. Apart from the unlikely case $\boldsymbol{\theta}=\theta_{Z},(10)$ clearly shows the oscillating structure of the product $\left(\boldsymbol{\theta}_{T} \cdot \boldsymbol{B}_{T}\right)$ which appears in (7) and which, for the photons considered above, reduces to the product $\theta_{z} B$. Then a clear signature of a nonzero $\boldsymbol{\theta}$ we are able to identify is a periodicity with frequency $\Omega$ in the number of pairs produced at fixed reaction plane which drastically changes during the earth rotation.

Putting everything together, we can assert here that, in the framework of NCED, the effects of a noncommutative spacetime can be detected in relativistic heavy ion collisions by the grid of the following experimental signatures/filtering procedures: (i) enhancement up to 10-20 per cent of small invariant mass lepton pairs coming from a very high energy photon with large transverse momentum in the reaction plane; (ii) the ratio of such pair production for nucleusnucleus collisions to the proton-proton case (A-B/p-p); (iii) time modulation of the produced pairs, due to the earth rotation.

A comment on the case of ultraperipheral collisions is in order before concluding. Indeed, in collisions with impact parameter larger than twice the nucleus radius, there is no competing process of pair production of partonic origin and the purely electromagnetic contribution, $\gamma \gamma \rightarrow e^{+} e^{-}$, is the standard background, strongly peaked in the low $p_{T}$ region. Any perturbative noncommutative correction to the previous process is negligible but a different, more relevant for our purpose, process could be the decay of a single noncommutative photon produced by bremsstrahlung. However, our threshold condition for pair production requires very large photon energies and a corresponding large suppression of the bremsstrahlung cross section. Therefore, we expect that it is difficult to isolate noncommutative effects in ultraperipheral collisions.

We then conclude that peripheral relativistic heavy ion collisions, where a huge magnetic field is produced, could be used to experimentally scrutinize radical hypotheses ${ }^{4}$ on the nature of spacetime we are long speculating about. In our opinion the search is worth the effort in any case, because if such effects would not be found one can nonetheless establish a new bound on the components of $\boldsymbol{\theta}$ associated with the oscillating factors, i.e. $\theta_{X}$ and $\theta_{Y}$, based solely on the kinematical features which characterize the decay of free

\footnotetext{
${ }^{4}$ Freeman Dyson in 2002, meeting one of us (A.I.) among researchers at MIT to hear about their work, defined as "radical" the hypothesis of a noncommutative spacetime.
} 
$\gamma$ into $e^{+} e^{-}$. At LHC for gold-gold collision the magnetic field could easily reach the intensity $B_{0} \simeq 3.2 \mathrm{GeV}^{2}$. If a free photon with an energy of $E_{\gamma} \simeq 100 \mathrm{GeV}$ travels in such magnetic field produced at the beginning of the collision and the pair production we described, i.e. points (i)-(iii), is not observed, then (7) gives the following bound:

$\theta_{X, Y}<\frac{2 m_{e}^{2}}{B E_{\gamma}^{2}} \simeq \frac{1}{10^{5}(\mathrm{TeV})^{2}} \simeq 10^{-3} \theta_{\mathrm{bound}}$

where $\theta_{\text {bound }}$ is the known bound [14].

Acknowledgements The authors thank D. Kharzeev and S. Voloshin for very useful discussions and suggestions.

Open Access This article is distributed under the terms of the Creative Commons Attribution Noncommercial License which permits any noncommercial use, distribution, and reproduction in any medium, provided the original author(s) and source are credited.

\section{References}

1. D.E. Kharzeev, Phys. Lett. B 633, 260 (2006)

2. D.E. Kharzeev, A. Zhitnitsky, Nucl. Phys. A 797, 67 (2007)

3. D.E. Kharzeev, L.D. McLerran, H.J. Warringa, Nucl. Phys. A 803, 227 (2008)

4. K. Fukushima, D.E. Kharzeev, H.J. Warringa, Real time dynamics of the Chiral magnetic effect. arXiv:1002.2495 [hep-ph]

5. B.I. Abelev et al. (Star Collaboration), Phys. Rev. Lett. 103, 251601 (2009)

6. B.I. Abelev et al. (Star Collaboration), Observation of chargedependent azimuthal correlations and possible local strong parity violation in heavy ion collisions. arXiv:0909.1717 [nucl-ex]

7. F. Wang, Effects of cluster particle correlations on local parity violation observables. arXiv:0911.1482 [nucl-ex]

8. R. Millo, E. Shuryak, Macroscopic chirality fluctuations in heavy ion collisions should induce $C P$ forbidden decays. arXiv:0912.4894 [hep-ph]

9. A. Bzdak, V. Koch, J. Liao, Remarks on possible local parity violation in heavy ion collisions. arXiv:0912.5050 [nucl-th]

10. V. Skokov, A.Y. Illarionov, V. Toneev, Int. J. Mod. Phys. A 24, 5925 (2009)
11. M.R. Douglas, N.A. Nekrasov, Rev. Mod. Phys. 73, 977 (2001)

12. R.J. Szabo, Phys. Rep. 378, 207 (2003)

13. I. Hinchliffe, N. Kersting, Y.L. Ma, Int. J. Mod. Phys. A 19, 179 (2004)

14. S.M. Carroll, J.A. Harvey, V.A. Kostelecky, C.D. Lane, T. Okamoto, Phys. Rev. Lett. 87, 141601 (2001)

15. A. Iorio, Phys. Rev. D 77, 048701 (2008)

16. A. Iorio, T. Sykora, Int. J. Mod. Phys. A 17, 2369 (2002)

17. D. Colladay, V. Kostelecky, Phys. Rev. D 58, 116002 (1998)

18. G. Amelino-Camelia, A perspective in quantum gravity phenomenology. arXiv:gr-qc/0402009

19. T. Jacobson, S. Liberati, D. Mattingly, Springer Proc. Phys. 98, 83 (2005)

20. F.W. Stecker, J. Phys. G 29, R47 (2003)

21. T. Jacobson, S. Liberati, D. Mattingly, Nature 424, 1019 (2003)

22. T. Jacobson, S. Liberati, D. Mattingly, F.W. Stecker, Phys. Rev. Lett. 93, 021101 (2004)

23. S. Minwalla, M. van Raamsdonk, N. Seiberg, J. High Energy Phys. 0002, 020 (2000)

24. A.P. Balachandran, A. Pinzul, B.A. Qureshi, Phys. Lett. B 634, 434 (2006)

25. Z. Guralnik, R. Jackiw, S.Y. Pi, A.P. Polychronakos, Phys. Lett. B 517, $450(2001)$

26. P. Castorina, A. Iorio, D. Zappalà, Phys. Rev. D 69, 065008 (2004)

27. P. Castorina, A. Iorio, D. Zappalà, Europhys. Lett. 69, 912 (2005)

28. P. Castorina, D. Zappalà, Europhys. Lett. 64, 641 (2003)

29. P. Castorina, A. Iorio, D. Zappalà, Nucl. Phys. B, Proc. Suppl. 136, 333 (2004)

30. J. Madore, S. Schraml, P. Schupp, J. Wess, Eur. Phys. J. C 16, 161 (2000)

31. N. Seiberg, E. Witten, J. High Energy Phys. 9909, 032 (1999)

32. O. Bertolami, et al., Phys. Rev. D 72, 025010 (2005)

33. D.E. Kharzeev, H.J. Warringa, Phys. Rev. D 80, 034028 (2009)

34. G. Baur, K. Hencken, D. Trautmann, Phys. Rep. 453, 1 (2007)

35. P. Aurenche, B. Baier, M. Fontannaz, Phys. Lett. B 209, 375 (1988)

36. S.A. Voloshin, Local strong parity violation and new possibilities in experimental study of non-perturbative QCD. arXiv:1003.1127 [nucl-ex]

37. F. Arleo et al., Writeup of the working group Photon Physics for the CERN Yellow Report on Hard Probes in Heavy Ion Collisions at the LHC. arXiv:hep-ph/0311131

38. A. Beddall, Eur. Phys. J. C 54, 365 (2008)

39. V.A. Kostelecky, C. Lane, Phys. Rev. D 60, 116010 (1999) 